\title{
Different fates of avermectin and artemisinin in China
}

\author{
Jinsong Chen ${ }^{1,5 \dagger}$, Jin Miao ${ }^{1 \dagger}$, Mei Liu ${ }^{1,5 \dagger}$, Xueting $\mathrm{Liu}^{1}$, Lianqun $\mathrm{Bao}^{2}$, Yuguo Jiang ${ }^{3}$, \\ Deming Wang ${ }^{3}$, Qing Zhang ${ }^{4 *} \&$ Lixin Zhang ${ }^{1,5 \ddagger}$ \\ ${ }^{1}$ Chinese Academy of Sciences Key Laboratory of Pathogenic Microbiology and Immunology, Institute of Microbiology, \\ Chinese Academy of Sciences, Beijing 100101; \\ ${ }^{2}$ Shijiazhuang Xingbai Bioengineering Co., Ltd, Shijiazhuang 050000; \\ ${ }^{3}$ Qilu Pharmaceutical Co., Ltd, Hohhot City 010080; \\ ${ }^{4}$ Inner Mongolia New Veyong Biochemical Co., Ltd, Dalad Banner 014300; \\ ${ }^{5}$ College of Life Sciences, University of Chinese Academy of Sciences, Beijing 100101
}

Received April 13, 2016; accepted April 22, 2016; published online April 28, 2016

Citation: Chen, J., Miao, J., Liu, M., Liu, X., Bao, L., Jiang, Y., Wang, D., Zhang, Q., and Zhang, L. (2016). Different fates of avermectin and artemisinin in China. Sci China Life Sci 59, 634-636. doi: 10.1007/s11427-016-5065-y

The 2015 Nobel Prize in Physiology or Medicine has been awarded to Youyou Tu, William C. Campbell and Satoshi Ōmura for the discoveries of artemisinin and avermectin. Their efficacy on parasitic disease treatment won the recognition of the whole world once again, revealing the dawning of the second "Golden age" in the development of natural products (http://dwz.cn/2u0RbE). Although artemisinin was originally innovated by Chinese, China holds neither proprietary intellectual property, nor unique leading technology or international market shares. In the case of avermectin, the situation is totally the opposite. With the forthcoming of the fourth industrial revolution, lessons learned from the success of avermectin may help transform China into a true powerhouse of innovation.

The breakthrough and advances in technologies and innovations correlate well with the rising up of the superpower nations in as seen throughout history. There have been three industrial revolutions since the mid-18th century. The first one is the age of water and steam power (1760-1840) that marks the transition from agricultural to industrial civilization. The second one is the age of electric power (1840-1950), an age leading to the boom of steel, railways, chemical engineering and automobiles. With the new ener-

\footnotetext{
†Contributed equally to this work

*Corresponding author (email: zhangqing@veyong.com)

†Corresponding author (email: zhanglixin@im.ac.cn)
}

gy provided by oil and with worldwide communications speeding up, the economy was pushed towards globalization. The third industrial revolution is the age of electronics and information technology, an age which began after World War II (1950-). The fourth industrial revolution (2006-) will fundamentally alter the way we live, work, and relate to each other. The ongoing synthetic biology guided by intelligent manufacturing covers the key achievements of digitization and networking.

Synthetic biology has bridged engineering principles with biotechnology, thus making biological design much easier. This development will enable a paradigm shift in the fermentation industry from the sole pursuit of production scale to the increase of efficiency and productivity of selected bacterial strains. Here, we review the history of the discovery of avermectin, the engineering of synthetic biology and the industrialization of avermectin in China. These three realities combine and provide guidance for a new era of "innovation in China".

In 1974, Professor Ōmura at Kitasato Institute isolated a new species of Streptomyces from a soil sample collected at Kawana, Ito City, Shizuoka Prefecture, Japan. This strain was later identified as Streptomyces avermitilis by Merck Sharp and Dohme Research Laboratories; the Ōmura group succeeded in identifying the structure of avermectin in 1975 from the fermentation broth of $S$. avermitilis. Dr. William 
C. Campbell discovered ivermectin, a derivative of avermectin, and highly effective against nematode parasites. From the 1980s, ivermectin has been widely applied in agriculture, animal husbandry and pharmaceutical industry. Thanks to these discoveries, river blindness, the human disease caused by Onchocerca volvulus was eradicated. This is also true of the horse disease $O$. cervicalis (Figure 1). Moreover, avermectin was found to show a synergistic effect with methicillin (MET) against methicillin-resistant Staphylococcus aureus (MRSA) (Guo et al., 2014) by a high-throughput synergy screening strategy (Zhang et al., 2007). These results shed light on potential therapeutics of anti-infective disease.

Before the discovery of avermectin biosynthetic gene clusters, the methods of fermentation optimization and random mutagenesis had been applied to improve avermectin production in the fermentation industry and the titer was increased from $0.009 \mathrm{~g} \mathrm{~L}^{-1}$ to $0.5 \mathrm{~g} \mathrm{~L}^{-1}$ (Siddique et al., 2013). After the genome of $S$. avermitilis was sequenced (Ikeda et al., 2003), a more rational method called metabolic engineering was introduced and widely used. For example, the overexpression of malEFG, which encodes a maltose ATP-binding cassette transporter, increases the supply of precursors by improving the utilization rate of starch ( $\mathrm{Li}$ et al., 2010). The overexpression of the ABC transporter AvtAB improves the efflux of avermectin (Qiu et al., 2011). The global regulator $\sigma^{H r d B}$ was identified as a key factor targeting the only positive regulator gene aveR that is located within the avermectin biosynthetic gene cluster. $\mathrm{Mu}-$ tant A56 with high-avermectin production was obtained by high-throughput screening of a $h r d B$ mutant library. This was further cultivated in a $180 \mathrm{~m}^{3}$ fermentor, and the production of avermectin B1a reached $6.38 \mathrm{~g} \mathrm{~L}^{-1}$ (Zhuo et al., 2010). The regulatory elements exploited in Streptomyces by the method of synthetic biology facilitate the design and engineering of avermectin biosynthetic pathway (Bai et al., 2015). In addition to this, other methods, such as the optimization of fermentation medium, inoculation technique, isolation and extraction technology, cyclic utilization of

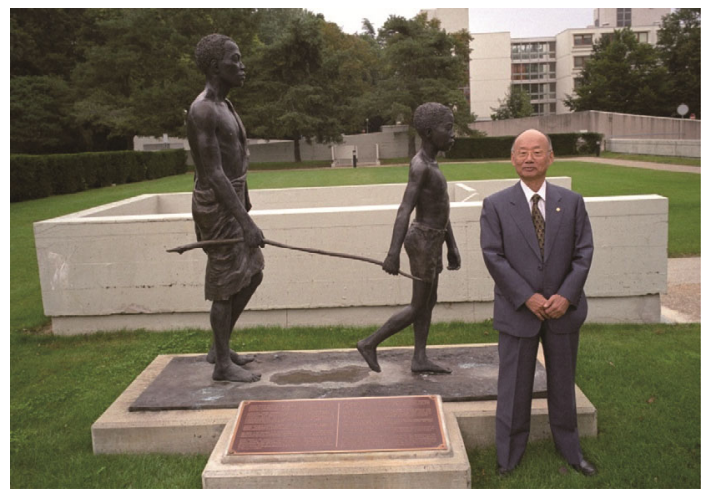

Figure 1 Professor Ōmura at WHO headquarters with the statue showing a boy leading a blind adult caused by $O$. volvulus infection. Professor Ōmura appreciates the contribution of Chinese scientists and pharmaceutical companies on the research and industry development of avermectin. wastewater from fermentation, and carbohydrate supplement process, not only increased the avermectin production, but at the same time also solved the problem of pollution from fermentation.

In China, studies on avermectin began in 1984. Due to the great contributions of Professors Yinchu Shen and Jilun $\mathrm{Li}$, large-scale industrial production was achieved. Professor Wen Liu did brilliant work on the optimization of components of avermectin. Professor Ying Wen systematically studied the regulatory network for avermectin biosynthesis. A breakthrough on efflux pumps for avermectin was made by Professor Linquan Bai. Professors Wensheng Xiang and Zhongjun Qin made significant contributions on the research and development of doramectin. Through collective efforts, researchers at the Institute of Microbiology Chinese Academy of Sciences (IMCAS) and their collaborators increased the production of avermectin B1a with a titer to $9 \mathrm{~g}$ $\mathrm{L}^{-1}$ and lowered the market price by forty times (Chen et al., 2016). In April 2010, the avermectin industrial alliance (AIA) composed of thirteen avermectin manufacturers was established. In January 2013, the National Basic Research Program of China "Fitness study on the synthetic microbial systems" was funded by the Ministry of Science and Technology (MOST). This study targeted the improvement of avermectin production using synthetic biology approaches. China is the only avermectin producing country in the world now with an annual sale above three billion RMB. In the interpretation of the 2015 Nobel Prize in Physiology or Medicine by Professor Hualiang Jiang (http://dwz.cn/ $2 \mathrm{u} 0 \mathrm{RbE}$ ), the contribution of IMCAS and AIA on avermectin industry, as well as the work unraveling the novel mechanism of artemisinin endoperoxide formation reaction (Yan et al., 2015), were emphasized. S. avermitilis was also transformed into a valuable chassis and cell factory for other natural products (Bai et al., 2015). Moving beyond the "world factory", China could become "a powerful fermentation country" with the help of synthetic and system biotechnology.

Compliance and ethics The author(s) declare that they have no conflict of interest.

Bai, C., Zhang, Y., Zhao, X., Hu, Y., Xiang, S., Miao, J., Lou, C., and Zhang, L. (2015). Exploiting a precise design of universal synthetic modular regulatory elements to unlock the microbial natural products in Streptomyces. Proc Natl Acad Sci USA 112, 12181-12186.

Chen J., Liu M., Liu X., Miao J., Fu C., Gao H., Müller R., Zhang Q., and Zhang L. (2016). Interrogation of Streptomyces avermitilis for efficient production of avermectins Synth Syst Biotechnol, doi: 10.1016/ j.synbio.2016.03.002.

Guo, H., Ren, B., Dai, H., Dai, S., Zhang, Y., Liu, Y., Cao, B., and Zhang, L. (2014). Reversal of meticillin resistance in Staphylococcus aureus by the anthelmintic avermectin. Int J Antimicrob Agents 44, 274-276.

Ikeda, H., Ishikawa, J., Hanamoto, A., Shinose, M., Kikuchi, H., Shiba, T., Sakaki, Y., Hattori, M., and Ōmura, S. (2003). Complete genome sequence and comparative analysis of the industrial microorganism 
Streptomyces avermitilis. Nat Biotechnol 21, 526-531.

Li, M., Chen, Z., Zhang, X., Song, Y., Wen, Y., and Li, J. (2010). Enhancement of avermectin and ivermectin production by overexpression of the maltose ATP-binding cassette transporter in Streptomyces avermitilis. Bioresour Technol 101, 9228-9235.

Qiu, J., Zhuo, Y., Zhu, D., Zhou, X., Zhang, L., Bai, L., and Deng, Z. (2011). Overexpression of the ABC transporter AvtAB increases avermectin production in Streptomyces avermitilis. Appl Microbiol Biotechnol 92, 337-345.

Siddique, S., Syed, Q., Adnan, A., Nadeem, M., Irfan, M., and Ashraf Qureshi, F. (2013). Production of Avermectin B1b From Streptomyces avermitilis 41445 by Batch Submerged Fermentation. Jundishapur J Microbiol 6.

Yan, W., Song, H., Song, F., Guo, Y., Wu, C., Sae Her, A., Pu, Y., Wang, S., Naowarojna, N., Weitz, A., Hendrich, M.P., Costello, C.E., Zhang,
L., Liu, P., and Zhang, Y. (2015). Endoperoxide formation by an alpha-ketoglutarate-dependent mononuclear non-haem iron enzyme. Nature 527, 539-543.

Zhang, L., Yan, K., Zhang, Y., Huang, R., Bian, J., Zheng, C., Sun, H., Chen, Z., Sun, N., An, R., Min, F., Zhao, W., Zhuo, Y., You, J., Song, Y., Yu, Z., Liu, Z., Yang, K., Gao, H., Dai, H., Zhang, X., Wang, J., Fu, C., Pei, G., Liu, J., Zhang, S., Goodfellow, M., Jiang, Y., Kuai, J., Zhou, G., and Chen, X. (2007). High-throughput synergy screening identifies microbial metabolites as combination agents for the treatment of fungal infections. Proc Natl Acad Sci USA 104, 4606-4611.

Zhuo, Y., Zhang, W., Chen, D., Gao, H., Tao, J., Liu, M., Gou, Z., Zhou, X., Ye, B.-C., Zhang, Q., Zhang, S., and Zhang, L.-X. (2010). Reverse biological engineering of hrdB to enhance the production of avermectins in an industrial strain of Streptomyces avermitilis. Proc Natl Acad Sci USA 107, 11250-11254.

Open Access This article is distributed under the terms of the Creative Commons Attribution License which permits any use, distribution, and reproduction in any medium, provided the original author(s) and source are credited. 